\title{
The clinical spectrum of CASQ1-related myopathy
}

Claudio Semplicini, MD, PhD, Cinzia Bertolin, PhD, Luca Bello, MD, PhD, Boris Pantic, PhD, Francesca Guidolin, MD, Sara Vianello, PhD, Francesco Catapano, PhD, Irene Colombo, MD, Maurizio Moggio, MD, Bruno F. Gavassini, PhD, Giovanna Cenacchi, MD, Valentina Papa, BSc, Marco Previtero, MD, Chiara Calore, MD, Gianni Sorarù, MD, PhD, Giovanni Minervini, PhD, Silvio C.E. Tosatto, PhD, Roberto Stramare, MD, and Elena Pegoraro, MD PhD

Neurology ${ }^{\circledR}$ 2018;91:e1629-e1641. doi:10.1212/WNL.0000000000006387

\section{Abstract}

\section{Objective}

To identify and characterize patients with calsequestrin 1 (CASQ1)-related myopathy.

\section{Methods}

Patients selected according to histopathologic features underwent CASQ1 genetic screening. CASQ1-mutated patients were clinically evaluated and underwent muscle MRI. Vacuole morphology and vacuolated fiber type were characterized.

\section{Results}

Twenty-two CASQ1-mutated patients (12 families) were identified, 21 sharing the previously described founder mutation (p.Asp244Gly) and 1 with the p.Gly103Asp mutation. Patients usually presented in the sixth decade with exercise intolerance and myalgias and later developed mild to moderate, slowly progressive proximal weakness with quadriceps atrophy and scapular winging. Muscle MRI $(\mathrm{n}=11)$ showed a recurrent fibrofatty substitution pattern. Three patients presented subclinical cardiac abnormalities. Muscle histopathology in patients with p.Asp244Gly showed vacuoles in type II fibers appearing empty in hematoxylin-eosin, Gomori, and nicotinamide adenine dinucleotide $(\mathrm{NADH})$ tetrazolium reductase stains but strongly positive for sarcoplasmic reticulum proteins. The muscle histopathology of p.Gly103Asp mutation was different, showing also $\mathrm{NADH}$-positive accumulation consistent with tubular aggregates.

\section{Conclusions}

We report the clinical and molecular details of the largest cohort of CASQ1-mutated patients. A possible heart involvement is presented, further expanding the phenotype of the disease. One mutation is common due to a founder effect, but other mutations are possible. Because of a paucity of symptoms, it is likely that CASQ1 mutations may remain undiagnosed if a muscle biopsy is not performed.

\author{
Correspondence \\ Dr. Pegoraro \\ elena.pegoraro@unipd.it
}




\section{Glossary}

BSA = bovine serum albumin; CASQ = calsequestrin; CK = creatine kinase; $\mathbf{N A D H}=$ nicotinamide adenine dinucleotide; ORAI1 = calcium release-activated calcium modulator 1 ; PBS = phosphate-buffered saline; SERCA1 = sarco/endoplasmic reticulum $\mathrm{Ca}^{2+}$ ATPase 1; SERCA2 = sarco/endoplasmic reticulum $\mathrm{Ca}^{2+}$ ATPase 2; SR = sarcoplasmic reticulum; STIM1 = stromal interaction molecule $1 ; \mathbf{T C}=$ terminal cisternae; $\mathbf{T R}=$ tetrazolium reductase.

$\mathrm{Ca}^{2+}$ transients are crucial in skeletal muscle excitationcontraction coupling. Synchronous $\mathrm{Ca}^{2+}$ release from the sarcoplasmic reticulum (SR) terminal cisternae (TC) into the cytoplasm and its reuptake into the SR store are granted by a structural and functional unit of $\mathrm{Ca}^{2+}$ sensors, release channels, buffering proteins, and ATP-dependent $\mathrm{Ca}^{2+}$ pumps at the junction of $\mathrm{T}$ tubule and TCs, i.e., the triad. ${ }^{1} \mathrm{~A}$ number of $\mathrm{Ca}^{2+}$-handling diseases involving the triad have been recently described, ${ }^{2}$ underlining the importance of its constituents in maintaining a correct $\mathrm{Ca}^{2+}$ homeostasis.

Calsequestrin (CASQ) represents the main $\mathrm{Ca}^{2+}$ buffer in the TC. ${ }^{3}$ The CASQ1 and CASQ2 isoforms are preferentially expressed in skeletal and cardiac/smooth muscle tissues, respectively, CASQ1 being largely predominant in fast-twitch (type II) fibers. ${ }^{4}$ CASQ1 buffers $\mathrm{Ca}^{2+}$ in the TC and prevents it from precipitating, thus maintaining a large-capacity gradient that can rapidly release large amounts of $\mathrm{Ca}^{2+}$ on stimulus. Despite this crucial role, the first human myopathy associated with CASQ1 mutations was only recently reported: a founder mutation (c.731A $>$ G, p.Asp244Gly) was first described in patients presenting a nondebilitating vacuolar myopathy, ${ }^{5,6}$ and other mutations were described in tubular aggregate myopathy, ${ }^{7,8}$

We report clinical and molecular characterization of the largest cohort of CASQ1-mutated patients, including 21 patients carrying the common p.Asp244Gly mutation and 1 carrying the p.Gly103Asp mutation. We expanded the clinical spectrum of the disease, identified a peculiar muscle MRI pattern, and further deepened molecular, immunohistochemical, and ultrastructural characterization of muscle biopsies, thus providing new details on the pathophysiologic aspects of this myopathy.

\section{Methods}

\section{Informed consent and ethics}

The study was conducted in accordance with the ethics rules and guidelines issued by local ethics committee and with the Declaration of Helsinki. Every patient provided written informed consent to study procedures.

\section{Patients}

All patients with unresolved vacuolar myopathy were selected from the database of patients referred to Padova Neuromuscular Center if they fulfilled the following inclusion criteria: vacuoles optically empty and/or containing amorphous material debris, no rimmed vacuoles, and no other causes of myopathy (i.e., normal muscular protein levels and acid maltase level; no exposure to chloroquine or colchicine).

\section{Genetic analyses}

Genomic DNA was extracted from peripheral blood by standard salting-out procedure. Tetra-primer Amplification Refractory Mutation System was designed to verify the presence of the CASQ1 c.731A $>\mathrm{G}$ variant, and positive results were confirmed by Sanger sequencing (primers and PCR conditions available on request). Total RNA was isolated from patients' skeletal muscle biopsies and reverse transcribed; CASQ1 cDNA (NM_001231) was amplified and Sanger sequenced. Any variation was checked against dbSNP, Exome Variant Server, 1000Genomes, and Genome Aggregation Database (Broad Institute, Cambridge, MA). Haplotype analysis was based on genotyping of 4 polymorphic 400-kb dinucleotide repeats around CASQ1.

\section{In silico investigation of p.Gly103Asp}

Sequence of human CASQ1 was retrieved from UniProt ${ }^{9}$ (identifier P31415), while orthologous sequences were collected from OMA Browser ${ }^{10}$ (accessed August 29, 2017). Sequences were visualized with Jalview. ${ }^{11}$ The mutation was modeled with Modeller ${ }^{12}$ using the wild-type crystal structure of CASQ16 (PDB code: $3 \mathrm{UOM}$, chain $\mathrm{A}$ ) as a template. The resulting model was used to perform molecular dynamics simulation to highlight the structural effect of the amino acid substitution. Pathogenicity of the p.Gly103Asp variant was assessed with a combination of 7 different predictors of protein stability, i.e., NeEMO, ${ }^{13}$ MUpro, ${ }^{14}$ Polyphen-2, ${ }^{15}$ SIFT, ${ }^{16}$ Pmut, ${ }^{17}$ SuSPect, ${ }^{18}$ and I-mutant $2.0 .^{19}$ Electrostatic properties were calculated with Bluues ${ }^{20}$ and visualized by means of Chimera. ${ }^{21}$ Secondary structure and sequence features were predicted with FELLS, ${ }^{22}$ while networks of interacting amino acids affected by mutations were calculated with RING 2.0. ${ }^{23}$ The overall fold stability was studied by performing $40 \mathrm{~ns}$ of molecular dynamics simulation with $\mathrm{NAMD}^{24}$ using the CHARMM $27^{25}$ force field. All simulation runs consisted of 100 conjugate gradient minimization steps, 100 ps in NVT conditions, 100 ps in NPT conditions, and 40 ns of classic molecular dynamics simulation. Temperature was kept at $300 \mathrm{~K}$, with pressure at $1.01325 \mathrm{bar}$, excluding NVT presimulation steps. The integration time step was set to 2 fs. PhospoSitePlus ${ }^{26}$ was used to predict phosphorylation sites.

\section{Clinical protocol}

The clinical features and progression of CASQ1-mutated patients were evaluated by revising medical records, structured questionnaires, and a specific evaluation protocol for motor, cardiac, and respiratory functions.

\section{Muscle imaging}

Patients underwent muscle MRI (1.5T, Avanto, Siemens, Erlangen, Germany) with T1-weighted axial scans. Analyzed 
muscles included pelvic, lower, and upper limb muscles. Degeneration and fatty infiltration were graded according to the modified Mercuri score. ${ }^{27} \mathrm{~A}$ final score ranging from 1 (normal) to 4 (severe involvement) was assigned to each muscle.

\section{Muscle histopathology and immunofluorescence}

Serial cryosections of muscle biopsies were stained with hematoxylin-eosin, ATPase, nicotinamide adenine dinucleotide $(\mathrm{NADH})$, and mouse monoclonal antibodies against calsequestrin (clone VIIID12; Thermo Fisher, Waltham, MA), ryanodine receptor, RYR1 (clone 34C; Abcam, Cambridge, UK), sarco/endoplasmic reticulum $\mathrm{Ca}^{2+}$ ATPase 1 (SERCA1; clone VE121G9; Santa Cruz Biotechnology, Santa Cruz, CA), stromal interaction molecule 1 (STIM1; clone 44/GOK; BD, San Jose, CA), rabbit polyclonal calcium release-activated calcium modulator 1, (ORAI1; Sigma-Aldrich Srl, Milan, Italy), and goat polyclonal sarco/endoplasmic reticulum $\mathrm{Ca}^{2+}$ ATPase 2 (SERCA2; N19; Santa Cruz). For immunofluorescence), muscle cryosections were collected on Superfrost slides, fixed with $4 \%$ paraformaldehyde for 10 minutes, permeabilized with $0.3 \%$ Triton X-100, incubated in blocking solution $(0.5 \%$ bovine serum albumin $[\mathrm{BSA}], 10 \%$ horse serum in phosphate-buffered saline $[\mathrm{PBS}])$ for 30 minutes, and incubated with antibodies at $4^{\circ} \mathrm{C}$ overnight ( $0.5 \% \mathrm{BSA}, 2 \%$ horse serum in PBS). Anti-goat Cy2-coniugated (Jackson Laboratory, Sacramento, CA), antimouse Cy3-coniugated, or anti-rabbit $\mathrm{Cy} 3$-coniugated (Invitrogen, Carlsbad, CA) secondary antibodies were incubated (0.5\% BSA, $2 \%$ horse serum in PBS) at room temperature for 1 hour. Slides were mounted with DAPI stain (Vector, Burlingame, CA) and examined in an upright microscope (Olympus BX60, Tokyo, Japan) equipped with a charge-coupled device camera (DP70, Olympus). Images were postprocessed with the Image suite. Single fibers were followed throughout serial sections, counted, and distinguished for the fiber type, presence of single or multiple vacuoles, and immunoreactivity of the vacuoles for the indicated antibodies. On average, 115 (range 40-240) fibers per patient were evaluated. Values were expressed as mean \pm SD percentage for the examined patients.

\section{Electron microscopy}

Small muscle specimens were fixed just after surgery in $2.5 \%$ glutaraldehyde in $0.1 \mathrm{~mol} / \mathrm{L}$ phosphate buffer at $\mathrm{pH} 7.2$ to 7.4 and postfixed in $1 \% \mathrm{OsO}_{4}$ in the same buffer. After dehydration in graded ethanol the specimens were embedded in Araldite. Thin sections were stained in uranyl acetate and lead citrate and observed in a Philips 410T transmission electron microscope.

\section{Statistical methods}

Median age at the onset of symptoms was estimated with the Kaplan-Meier method. Frequency of vacuolated fibers was compared by the Fisher exact test. Statistical significance was set at $p<0.05$.

\section{Data availability}

Anonymized data will be shared by request from any qualified investigator.

\section{Results}

\section{CASQ1 mutation analyses}

Forty-seven unrelated patients were tested for presence of the c.731A>G (p.Asp244Gly) variant. The mutation was identified in 10 patients in heterozygosis. The collection of detailed family history information identified 7 additional affected family members. Four already reported patients (patients 9-12 ${ }^{5}$ ) were also included. Overall, we identified the common p.Asp244Gly mutation in 21 patients belonging to 12 unrelated pedigrees. A common haplotype was shared by all p.Asp244Gly alleles, confirming the known founder effect. $^{5}$

CASQ1 sequencing was performed in 37 patients negative for the p.Asp244Gly variant and identified in 1 patient the heterozygous variant c.308G $>$ A (p.Gly103Asp).

\section{In silico investigation of p.Gly103Asp}

Sequence conservation analysis shows Gly103 to be fully conserved among mammals (figure 1, A). This residue is located in a mostly conserved large region, forming a $\left(\mathrm{Ca}^{2+}\right)$ independent high-affinity $\mathrm{Ca}^{2+}$-binding site. The mutation was predicted to decrease protein stability, possibly introducing a local unfold (figure 1, B), by most predictors. Pmut ${ }^{17}$ predicted a putative neutral effect. CASQ1 is mostly acidic with several negative charged residues, so the introduction of a further negative charge may induce local unfolding driven by electrostatic repulsion. Previous evidence showed p.Gly103Asp to be more susceptible to trypsin proteolysis, ${ }^{8}$ suggesting this mutation to have a destabilizing effect on protein structure. To shed light on these findings, we performed fine electrostatic change analysis coupled with molecular dynamics simulations. Predictions with Bluues ${ }^{20}$ showed p.Gly103Asp to induce a relevant electrostatic change (figure 1, C). In particular, Gly103 is close to Asp65, and we believe that the electrostatic repulsion may trigger a local backbone reorganization. This finding is in agreement with molecular dynamics simulations (figure 1, C). The p.Gly103Asp mutation is predicted to induce unfolding of an a-helix (residues 78-102). Furthermore, Gly103 forms a bend connecting 2 structured elements, and substitution with Asp is predicted to induce a local structural rearrangement. Inspection with RING ${ }^{23}$ confirms the repulsive interaction between p.Gly103Asp and Asp65. Of note, the $\alpha$-helix, which is predicted to unfold, is in contact with a putative phosphorylation site, i.e., Tyr43. A similar destabilizing effect is also predicted by FELLS, ${ }^{22}$ which predicts an increased tendency to form intrinsically disordered conformations in this region (figure 1, D).

\section{Clinical evaluation}

Twenty-two CASQ1-mutated patients were included (18 male, 4 female patients) (table). Age at last evaluation ranged from 16 to 83 years (mean $51.5 \pm 18.5$ years). Ten patients 29 to 71 years of age were asymptomatic, and the diagnosis was posed because of asymptomatic hyperCKemia or family 


\section{A}

H. Sapiens 88 EELILELAAQVLEDKGVGFGLVDSEKDAAVA 118

O. Cuniculus 82 EELILELAAQVLEDKGVGFGLVDSEKDAAVA 112

M. Musculus 88 EELILELAAQVLEDKGVGFGLVDSEKDAAVA 118

R. Norvegicus 88 EELILELAAQVLEDKGVGFGLVDSEKDAAVA 118

B. Taurus 88 DELILELAAQVLEDKGVGFGMVDSEKDAAVA 118

O. Aries 88 EELILELAAQVLEDKGVGFGMVDSEKDAAVA 118

S. Scrofa 88 EELILELAAQVLEDKGVGFGMVDSEKDAAVA 118

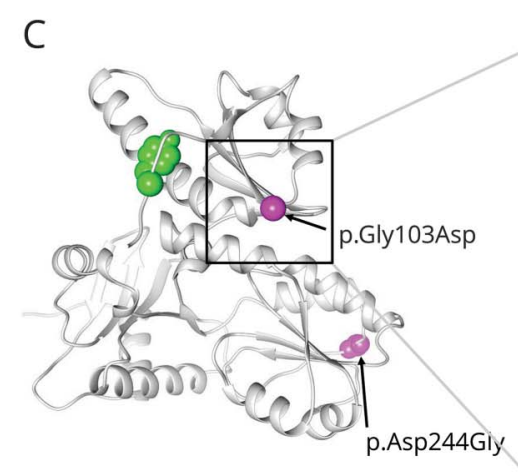

B

$\begin{array}{ccc}\text { Predictor } & \text { Stability change } & \begin{array}{c}\text { Confidence } \\ \text { score }\end{array} \\ \text { NeEMO } & \text { Decrease } & \\ \text { MUpro SVM } & \text { Decrease } & -0.37 \\ \text { MUpro NN } & \text { Decrease } & -0.94 \\ \text { PolyPhen-2 } & \text { Possibly damaging } & \\ \text { SIFT } & \text { Tolerated } & 3.08 \\ \text { Pmut } & \text { Neutral } & 0.11 \\ \text { SuSPect } & \text { Possibly damaging } & \\ \text { I-Mutant 2.0 } & \text { Decrease } & \end{array}$

\section{D}

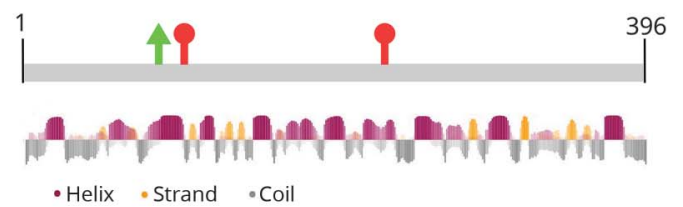

(A) Multiple sequence alignment of calsequestrin 1 (CASQ1) ortholog proteins from different mammals. (B) Stability prediction assessed with different tools. The predicted stability change is reported as predicted by the different tools, with a confidence score reported when available. (C) Cartoon representation of human CASQ1. Magenta spheres represent the position of mutations, and green is used for the putative phosphorylation site. Electrostatic change and molecular dynamics simulation are presented in the box. (D) Sequence feature and secondary structure prediction. Gray, purple, and yellow fells represent disorder, a-helix, and $\beta$-sheet, respectively. WT = wild type.

history. Median age at the onset of symptoms was 65 years (95\% confidence interval 54-undetermined) (figure 2). Onset symptoms included myalgia (6 cases), exercise intolerance (5), pelvic girdle weakness (4), and falls (2). Of note, 3 patients reported minimal symptoms since childhood (running and sport difficulties, scoliosis). Creatine kinase (CK) levels at disease onset or before first symptoms were elevated at rest in all but 1 patient, ranging from 1 to 75 times the upper limit of normal (median 5.5). Exertional rhabdomyolysis was reported in 3 cases. No cases of malignant hyperthermia were reported.

Manual muscle testing showed mild to moderate weakness in 10 of 20 patients, more frequently in hip flexors (8 cases), triceps brachii (7), scapular muscles (6), knee flexors (4), neck flexors (3), deltoid (3), and biceps brachii (2). Scapular winging (8 cases), quadriceps atrophy (4), or pectoral atrophy and distal lower limb atrophy (3) were frequent. Knee deep tendon reflexes were brisk in 9 of 20 patients. Two patients presented mild knee and elbow contractures. Respiratory evaluation (8 patients) showed a mild decrease of respiratory muscle strength in 2 patients (patients 1 and 2) and mild chronic obstructive pulmonary disease in 1 patient (patient 9). Echocardiography (performed in 12 patients) showed in patient 17 a left ventricular hypertrophy predominantly at the septal level, not related to hypertension, with minimal signs of fibrosis, confirmed by cardiac MRI. Two other patients presented subclinical echocardiographic abnormalities, i.e., a reduction in longitudinal strain in an asymptomatic 18year-old (patient 20) and hypertensive heart disease in a 79-year-old man (patient 9).

\section{Muscle imaging}

Eleven patients (age range 16-79 years) underwent muscle MRI (figure 3). Four patients (aged 16-37 years) showed normal T1-weighted sequences. In the others, a peculiar pattern of fibrofatty substitution was observed, with a correlation between severity of degeneration and age. Muscle alterations appeared first in the distal portion of the vastus lateralis, in the semitendinosus, and in the gluteus maximus. With increasing age, fibrofatty substitution spread to all anterior thigh muscles, with relative sparing of vastus intermedius; to posterior thigh muscles, with relative sparing of sartorius and proximal biceps femoris; and to the posterior compartment of the leg, especially in the gastrocnemius. Of note, remarkable asymmetry was observed in all patients. In the upper limbs, only the 2 oldest patients presented mild involvement of triceps brachii ( 2 of 2), deltoid, and subscapularis (1 of 2$)$.

\section{Histopathology and immunofluorescence studies}

Serial cryosections of 9 p.Asp244Gly-mutated patients and 1 p.Gly103Asp-mutated patient were analyzed. An average of $116 \pm 59$ (SD) fibers were analyzed per patient. As described, ${ }^{5}$ p.Asp244Gly resulted in the appearance of optically empty vacuoles in hematoxylin-eosin and acidic ATPase. We also 


\section{Table Clinical features of CASQ1-mutated patients}

\begin{tabular}{|c|c|c|c|c|c|c|c|c|c|c|c|c|c|}
\hline \multirow[b]{2}{*}{$\begin{array}{l}\text { Patient } \\
\text { n., sex }\end{array}$} & \multirow[b]{2}{*}{$\begin{array}{l}\text { Family } \\
\text { No. }\end{array}$} & \multicolumn{3}{|l|}{ Disease onset } & \multicolumn{9}{|c|}{ Disease course } \\
\hline & & $\begin{array}{l}\text { Age at first } \\
\text { symptoms, y }\end{array}$ & $\begin{array}{l}\text { First } \\
\text { symptoms }\end{array}$ & $\begin{array}{l}\text { CK at } \\
\text { onset } \\
(\times \\
\text { n.v. })\end{array}$ & $\begin{array}{l}\text { Age at last } \\
\text { evaluation, } \\
\text { y }\end{array}$ & $\begin{array}{l}\text { Upper } \\
\text { limb } \\
\text { muscular } \\
\text { weakness }\end{array}$ & $\begin{array}{l}\text { Lower } \\
\text { limb } \\
\text { muscular } \\
\text { weakness }\end{array}$ & $\begin{array}{l}\text { Axial } \\
\text { muscles } \\
\text { weakness }\end{array}$ & Muscle trophism & $\begin{array}{l}\text { Deep } \\
\text { reflexes }\end{array}$ & $\begin{array}{l}\text { Cardiac } \\
\text { function }\end{array}$ & $\begin{array}{l}\text { Respiratory } \\
\text { function }\end{array}$ & $\begin{array}{l}\text { Associated } \\
\text { pathologies }\end{array}$ \\
\hline $1, F$ & $1 a$ & 34 & $\begin{array}{l}\text { Muscle pain, } \\
\text { frequent falls }\end{array}$ & $\times 1.5$ & 52 & $\begin{array}{l}\text { Triceps } \\
\text { brachii; } \\
\text { scapular } \\
\text { muscles }\end{array}$ & $\begin{array}{l}\text { Knee } \\
\text { flexors, hip } \\
\text { flexors }\end{array}$ & $\begin{array}{l}\text { Neck } \\
\text { flexor }\end{array}$ & Scapular winging & $\begin{array}{l}\text { Knee } \\
\text { reflexes } \\
\text { very } \\
\text { brisk }\end{array}$ & Normal & $\begin{array}{l}\text { Mild } \\
\text { respiratory } \\
\text { muscle } \\
\text { weakness }\end{array}$ & Hemicrania \\
\hline $2, \mathrm{M}$ & $1 \mathrm{~b}$ & 65 & Muscle pain & $\begin{array}{l}\times 6 \\
(\times 10 \\
\text { after } \\
\text { effort })\end{array}$ & 67 & $\begin{array}{l}\text { Triceps } \\
\text { brachii; } \\
\text { scapular } \\
\text { muscles }\end{array}$ & $\begin{array}{l}\text { Hip flexors, } \\
\text { ankle } \\
\text { dorsiflexion }\end{array}$ & No & $\begin{array}{l}\text { Quadriceps } \\
\text { hypotrophy }\end{array}$ & Absent & Normal & $\begin{array}{l}\text { Mild } \\
\text { inspiratory } \\
\text { muscles } \\
\text { weakness }\end{array}$ & $\begin{array}{l}\text { Hypertension, } \\
\text { Dupuytren } \\
\text { syndrome }\end{array}$ \\
\hline $3, M$ & $1 b$ & 70 & $\begin{array}{l}\text { Exercise } \\
\text { intolerance, } \\
\text { lower limb } \\
\text { proximal } \\
\text { weakness }\end{array}$ & $\times 4$ & 83 & NA & NA & NA & Normal & Brisk & Normal & Normal & $\begin{array}{l}\text { Hypertension, } \\
\text { sensorineural } \\
\text { hearing loss }\end{array}$ \\
\hline $4, M$ & $1 b$ & 45 & $\begin{array}{l}\text { Exercise } \\
\text { intolerance }\end{array}$ & $\times 7$ & 55 & No & No & No & $\begin{array}{l}\text { Quadriceps } \\
\text { hypotrophy }\end{array}$ & Brisk & & & $\begin{array}{l}\text { Sensorineural } \\
\text { hearing loss }\end{array}$ \\
\hline $5, M$ & 2 & 47 & $\begin{array}{l}\text { Exercise } \\
\text { intolerance, } \\
\text { muscle pain }\end{array}$ & $\times 7$ & 60 & $\begin{array}{l}\text { Triceps } \\
\text { brachii }\end{array}$ & $\begin{array}{l}\text { Knee } \\
\text { flexors, hip } \\
\text { flexors }\end{array}$ & No & $\begin{array}{l}\text { Quadriceps } \\
\text { hypotrophy, } \\
\text { scapular winging }\end{array}$ & Brisk & & & Hypertension \\
\hline $6, F$ & 2 & $\begin{array}{l}\text { Asymptomatic } \\
\text { (35) }\end{array}$ & HyperCKemia & $\times 11$ & 37 & No & No & No & $\begin{array}{l}\text { Mild scapular } \\
\text { winging }\end{array}$ & Brisk & & & \\
\hline $7, \mathrm{M}$ & 3 & $\begin{array}{l}\text { Asymptomatic } \\
\text { (37) }\end{array}$ & HyperCKemia & $\begin{array}{l}\times 11 \\
(\times 27 \\
\text { after } \\
\text { effort })\end{array}$ & 37 & No & No & No & Normal & Brisk & & & \\
\hline $8, M$ & 4 & 43 & $\begin{array}{l}\text { Exercise } \\
\text { intolerance }\end{array}$ & $\times 5$ & 61 & No & No & No & Normal & Normal & Normal & & $\begin{array}{l}\text { Nonprogressive } \\
\text { demyelinating } \\
\text { encephalopathy }\end{array}$ \\
\hline $9, M$ & 5 & 65 & $\begin{array}{l}\text { Frequent falls, } \\
\text { mild lower } \\
\text { limb proximal } \\
\text { weakness }\end{array}$ & $\times 4$ & 79 & $\begin{array}{l}\text { Biceps } \\
\text { brachii, } \\
\text { triceps } \\
\text { brachii }\end{array}$ & $\begin{array}{l}\text { Knee } \\
\text { flexors, hip } \\
\text { flexors, } \\
\text { knee } \\
\text { extensor }\end{array}$ & No & $\begin{array}{l}\text { Scapular winging, } \\
\text { muscle hypotrophy } \\
\text { of quadriceps, } \\
\text { pectoral, distal leg }\end{array}$ & Reduced & $\begin{array}{l}\text { Hypertensive } \\
\text { heart disease }\end{array}$ & $\begin{array}{l}\text { Mild chronic } \\
\text { obstructive } \\
\text { pulmonary } \\
\text { disease }\end{array}$ & $\begin{array}{l}\text { Chronic } \\
\text { obstructive } \\
\text { pulmonary } \\
\text { disease }\end{array}$ \\
\hline $10, M$ & 5 & $\begin{array}{l}\text { Asymptomatic } \\
\text { (35) }\end{array}$ & HyperCKemia & $\begin{array}{l}\times 2 \\
(\times 21 \\
\text { after } \\
\text { effort })\end{array}$ & 35 & No & No & No & Normal & $\begin{array}{l}\text { Very } \\
\text { brisk }\end{array}$ & Normal & Normal & $\begin{array}{l}\text { Multiple } \\
\text { sclerosis }\end{array}$ \\
\hline
\end{tabular}


Table Clinical features of CASQ1-mutated patients (continued)

\begin{tabular}{|c|c|c|c|c|c|c|c|c|c|c|c|c|c|}
\hline \multirow[b]{2}{*}{$\begin{array}{l}\text { Patient } \\
\text { n., sex }\end{array}$} & \multirow[b]{2}{*}{$\begin{array}{l}\text { Family } \\
\text { No. }\end{array}$} & \multicolumn{3}{|l|}{ Disease onset } & \multicolumn{9}{|c|}{ Disease course } \\
\hline & & $\begin{array}{l}\text { Age at first } \\
\text { symptoms, y }\end{array}$ & $\begin{array}{l}\text { First } \\
\text { symptoms }\end{array}$ & $\begin{array}{l}\text { CK at } \\
\text { onset } \\
(\times \\
\text { n.v. })\end{array}$ & $\begin{array}{l}\text { Age at last } \\
\text { evaluation, } \\
\text { y }\end{array}$ & $\begin{array}{l}\text { Upper } \\
\text { limb } \\
\text { muscular } \\
\text { weakness }\end{array}$ & $\begin{array}{l}\text { Lower } \\
\text { limb } \\
\text { muscular } \\
\text { weakness }\end{array}$ & $\begin{array}{l}\text { Axial } \\
\text { muscles } \\
\text { weakness }\end{array}$ & Muscle trophism & $\begin{array}{l}\text { Deep } \\
\text { reflexes }\end{array}$ & $\begin{array}{l}\text { Cardiac } \\
\text { function }\end{array}$ & $\begin{array}{l}\text { Respiratory } \\
\text { function }\end{array}$ & $\begin{array}{l}\text { Associated } \\
\text { pathologies }\end{array}$ \\
\hline $11, M$ & 6 & $\begin{array}{l}\text { Asymptomatic } \\
\text { (69) }\end{array}$ & HyperCKemia & NA & 69 & $\begin{array}{l}\text { Deltoid, } \\
\text { scapular } \\
\text { muscles, } \\
\text { triceps } \\
\text { brachii }\end{array}$ & No & No & Scapular winging & Normal & & & Ulcerative colitis \\
\hline $12, \mathrm{M}$ & 6 & $\begin{array}{l}\text { Asymptomatic } \\
\text { (33) }\end{array}$ & HyperCKemia & $\times 12$ & 33 & No & No & No & Scapular winging & Normal & Normal & & \\
\hline $13, M$ & 6 & 54 & $\begin{array}{l}\text { Mild proximal } \\
\text { weakness }\end{array}$ & $\times 5$ & 57 & $\begin{array}{l}\text { Deltoid, } \\
\text { triceps } \\
\text { brachii }\end{array}$ & Hip flexors & No & NA & NA & & & \\
\hline $14, M$ & 7 & NA & $\begin{array}{l}\text { Exercise } \\
\text { intolerance, } \\
\text { muscle pain }\end{array}$ & NA & 52 & NA & NA & NA & NA & NA. & & & \\
\hline $15, M$ & 8 & 15 & $\begin{array}{l}\text { Mild proximal } \\
\text { weakness }\end{array}$ & $\times 11$ & 16 & No & Hip flexors & No & $\begin{array}{l}\text { Pectoral and distal } \\
\text { leg muscles } \\
\text { hypotrophy }\end{array}$ & Normal & & & \\
\hline $16, M$ & 8 & $\begin{array}{l}\text { Asymptomatic } \\
\text { (59) }\end{array}$ & HyperCKemia & $\times 4$ & 59 & No & No & No & $\begin{array}{l}\text { Pectoral and distal } \\
\text { leg muscle } \\
\text { hypotrophy }\end{array}$ & Normal & & & \\
\hline $17, \mathrm{M}$ & 9 & $\begin{array}{l}\text { Asymptomatic } \\
\text { (59) }\end{array}$ & HyperCKemia & $\times 3$ & 59 & No & No & No & Normal & Normal & $\begin{array}{l}\text { Left } \\
\text { ventricular } \\
\text { hypertrophy }\end{array}$ & Normal & \\
\hline $18, F$ & 9 & $\begin{array}{l}\text { Asymptomatic } \\
\text { (63) }\end{array}$ & HyperCKemia & $\times 1$ & 64 & $\begin{array}{l}\text { Deltoid, } \\
\text { scapular } \\
\text { muscles, } \\
\text { triceps, } \\
\text { biceps }\end{array}$ & Hip flexors & $\begin{array}{l}\text { Neck } \\
\text { flexor }\end{array}$ & Scapular winging & Brisk & & & \\
\hline $19, \mathrm{M}$ & 9 & $\begin{array}{l}\text { Asymptomatic } \\
\text { (29) }\end{array}$ & HyperCKemia & $\times 13$ & 29 & No & No & No & Normal & Normal & $\begin{array}{l}\text { Normal } \\
\text { (longitudinal } \\
\text { strain in lower } \\
\text { normal limits) }\end{array}$ & & Epilepsy \\
\hline $20, M$ & 10 & 12 & Muscle pain & $\times 75$ & 18 & No & No & No & Normal & Normal & $\begin{array}{l}\text { Strain } \\
\text { reduction }\end{array}$ & Normal & \\
\hline $21 \mathrm{M}$ & 11 & 39 & Muscle pain & $\times 11.5$ & 40 & $\begin{array}{l}\text { Scapular } \\
\text { muscles }\end{array}$ & $\begin{array}{l}\text { Knee } \\
\text { flexors, hip } \\
\text { flexors }\end{array}$ & No & Scapular winging & Brisk & Normal & & \\
\hline
\end{tabular}


Figure 2 Cumulative incidence plot of the onset of symptoms

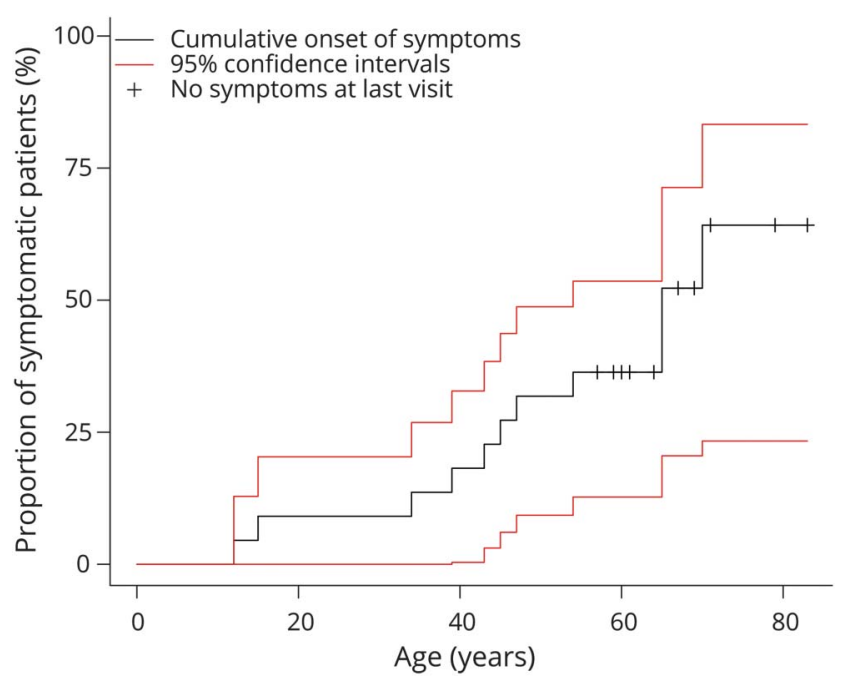

Muscle weakness, myalgia, or exercise intolerance in 22 calsequestrin 1 (CASQ1)-mutated patients. 95\% Confidence intervals are indicated as dashed lines. Asymptomatic patients are marked by a censoring symbol (+) at the age at last evaluation.

observed an increased variation in fiber size diameter $(6-86 \mu \mathrm{m})$, rare necrotic fibers, central nuclei, splitting, and acid phosphatase activation in scattered fibers. Immunofluorescence analysis of sarcoplasmic $\mathrm{Ca}^{2+}$ influx/efflux machinery proteins showed reactivity for CASQ1, RYR1, ORAI1, SERCA1, and STIM1 at the edge of the vacuoles. Vacuoles were observed almost exclusively in type II fibers (204 of 488 type II fibers vs 4 of 557 type I fibers, $p<0.001$ ) and most of the time were multiple ( $82 \pm 14 \%$ of all vacuolated fibers). CASQ1 and SERCA1 displayed the highest levels of enrichment, which was observed in $86 \pm 16 \%$ and $74 \pm 20 \%$ of type II vacuolated fibers, respectively, while lower levels of enrichment on vacuole margins were observed for ORAI1, RYR1, and STIM1, which were detected in $72 \pm 30 \%, 60 \pm 23 \%$, and $57 \pm 33 \%$, respectively. The weakest increase in signal and the smallest frequency of enrichment of vacuoles were seen for SERCA2, normally expressed at low levels in fast-twitch fibers ( $38 \pm 18 \%$ of vacuolated fibers) (figure 4).

The p.Gly103Asp mutation resulted in a different muscle morphology, showing vacuoles filled with amorphous material (in 15 of 62 fibers, type II only) that appeared red on Gomori trichrome and dark blue on NADH tetrazolium reductase (NADH-TR) staining and were negative with succinic-dehydrogenase staining, consistent with tubular aggregates (figures 4 and 5). Unfortunately, ultrastructural analysis was not performed because of tissue unavailability. No optically empty vacuoles were observed. The intrafiber vacuoles were strongly immunoreactive for CASQ1, ORAI1, RYR1, SERCA1, and STIM1 and weakly immunoreactive for SERCA2 (figure 4). 
A
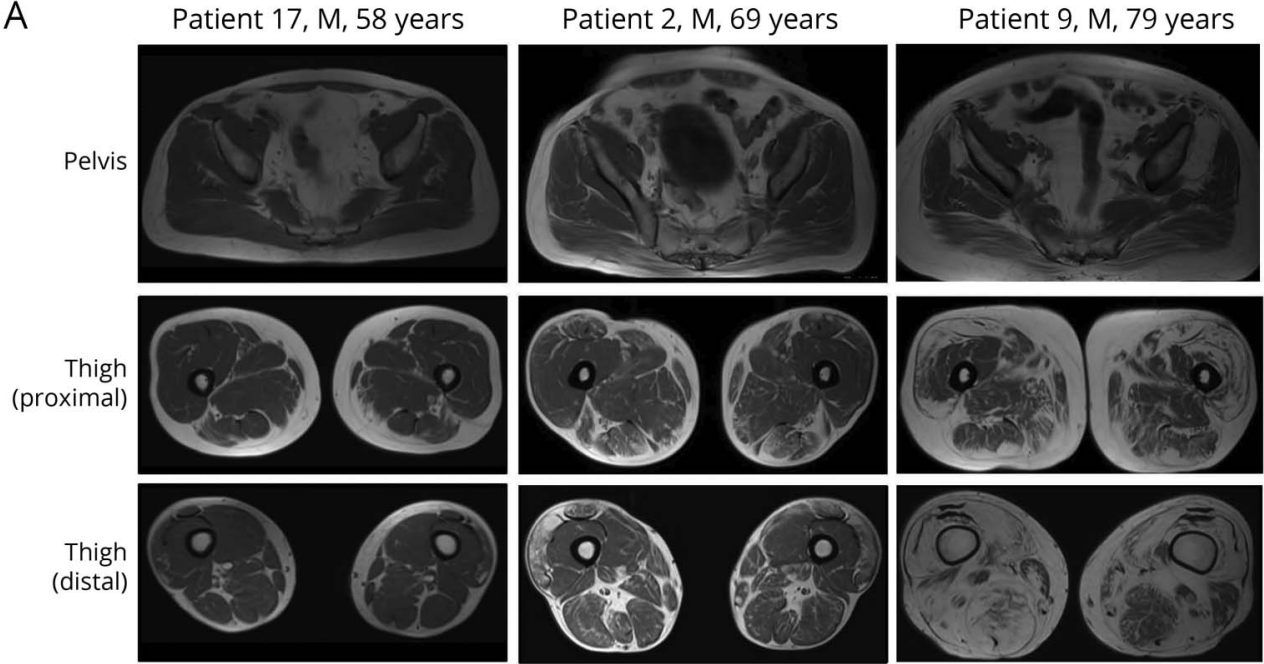

Leg
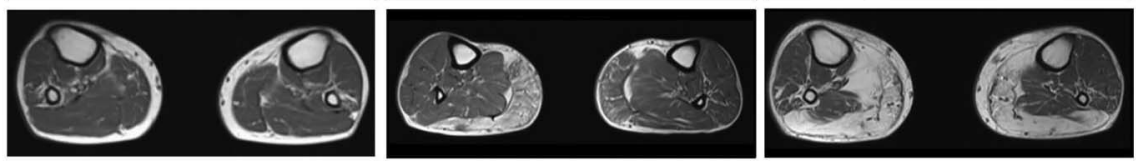

B

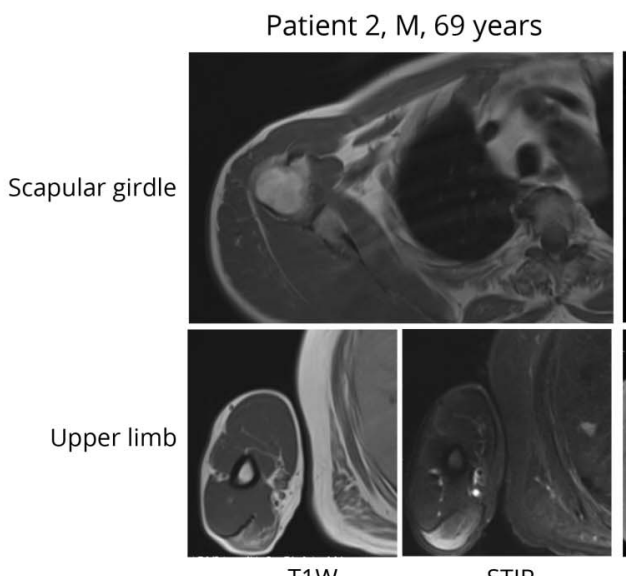

T1W

STIR

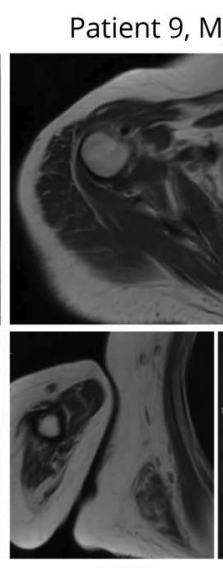

T1W
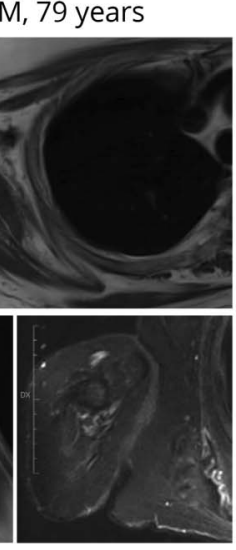

STIR
(A) Lower limb muscle MRI. T1 weighted (T1W) sequences in 3 patients of different ages. Age-dependent and asymmetric degree of fibrofatty substitution can be observed initially in the distal portion of vastus lateralis, semitendinosus muscle, and gluteus maximus, late spreading to all anterior thigh muscles with relative sparing of the vastus intermedius and to posterior thigh muscles with relative sparing of sartorius and proximal portion of biceps femoris. A severe involvement of gastrocnemius medialis is shown. (B) Scapular and upper limb muscle MRI. T1W sequences in the 2 oldest patients show moderate involvement of triceps brachii.

\section{Electron microscopy}

Ultrastructural analysis was able to dissect the mechanisms of accumulation of electron-dense polygonal structures in p.Asp244Gly-mutated specimens (figure 6). Various stages of ultramicroscopic alterations were identified. In the early stages, areas of myofibrillar lysis (figure 6, A) seemed to be replaced by a progressive accumulation of glycogen granules (figure 6, B), followed by the appearance of triangular and/or quadrangular electron-dense, sharp-margined inclusions, with outlines resembling puzzle pieces (figure 6, C). Geometric inclusions appear to be outlined by a thin layer of glycogen particles. Mitochondrial aggregates with normal morphology could be detected. Some of the muscle biopsies showed SRderived tubular aggregates detectable mainly in the subsarcolemmal area (figure 6, D).

\section{Discussion}

Recently, CASQ1 has been established as a muscle disease gene. $^{5-8,28}$ CASQ1-related myopathy is clinically characterized by high CK levels, mild proximal weakness, and myalgia. In this study, we further defined the clinical spectrum of the disease.

The CASQ1 genetic screening in unresolved vacuolar myopathies confirmed a high prevalence of CASQ1 mutations (12 of 49, 24\%). The identification of the p.Asp244Gly mutation in 21 patients confirmed a founder effect possibly arising in the Padova district area. The recently described p.Gly103Asp mutation $^{7,8}$ was detected in an additional patient hailing from central Italy (Tuscany) who had a tubular aggregate myopathy. 


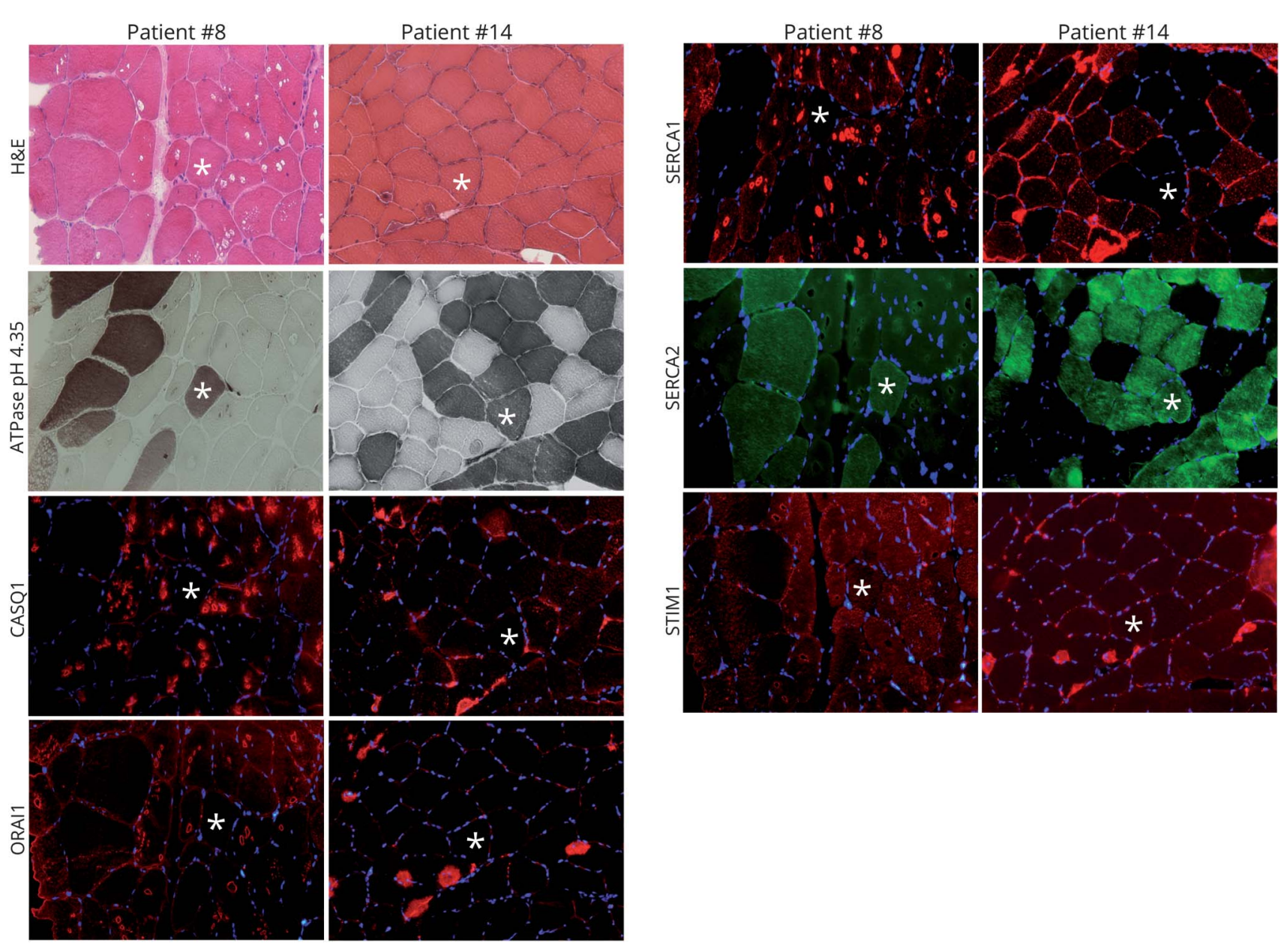

Serial cryosections of p.Asp244Gly (patient 8) and p.Gly103Asp (patient 14) muscle biopsy (all panels original magnification ×20). In patient 8, in hematoxylineosin (H\&E) optically empty vacuoles almost exclusively in type II fibers, increased fiber size variation, central nuclei, and splitting are shown. Immunofluorescence analysis of sarcoplasmic $\mathrm{Ca}^{2+}$ influx/efflux machinery proteins shows reactivity for calsequestrin 1 (CASQ1), ORAI1, SERCA1, and STIM1 at the edge of the vacuoles that appear optically empty with acidic ATPase. In patient 14, vacuoles filled with amorphous material (H\&E) corresponding to tubular aggregates are shown. No optically empty vacuoles are detected. The intrafiber vacuoles are strongly immunoreactive for CASQ1, ORAI1, SERCA1, and STIM1 and weakly immunoreactive for SERCA2.

In our case series, the clinical features are homogeneous. The onset typically occurs around the sixth decade with relatively mild symptoms (myalgia, exercise intolerance, or mild proximal weakness). HyperCKemia is common and sometimes the unique clinical sign leading to diagnosis, suggesting that CASQ1-related myopathies may be underdiagnosed. The clinical progression of CASQ1 myopathy is usually slow. However, some patients presented with unrecognized clinical symptoms since a very young age, indicating a very early and subtle onset, while some older patients presented with severely disabling muscular weakness. Muscle MRI studies further confirmed a progression toward severe fibrofatty substitution. The recurrence of rhabdomyolysis in 3 cases (marked CK increase and postexertional myalgia) and the presence of exercise intolerance indicate that CASQ1 myopathies may also present as a pseudometabolic myopathy with dynamic symptoms instead of fixed weakness.
Muscular weakness was relatively mild and age dependent and mostly involved triceps brachii, scapular muscles, hip flexors, and knee flexors. We frequently observed muscle atrophy involving the quadriceps, scapular muscles, or pectoral muscles. This is different from previously described cohorts, ${ }^{5,6,8}$ in which patients sometimes presented with mild hypertrophy. An accurate neurologic examination showed brisk knee reflexes in 9 of 20 patients, a sign not frequently observed in myopathic patients with quadriceps involvement. This feature could indicate a possible role of CASQ1 in the CNS. Indeed, CASQ1 is also expressed in nonexcitable tissues, where it plays a role in buffering endoplasmic reticulum calcium and in calcium release. ${ }^{29,30}$ There is increasing evidence that CASQ1 is expressed in the cerebral cortex (the Human Protein Atlas ${ }^{31}$ ) where it might be enriched in discrete brain areas, as previously described for CASQ2. 32,33 Hypothetically, a CASQ1-mutated protein in upper motor neuron may be responsible for brisk reflexes observed in patients. Curiously, 

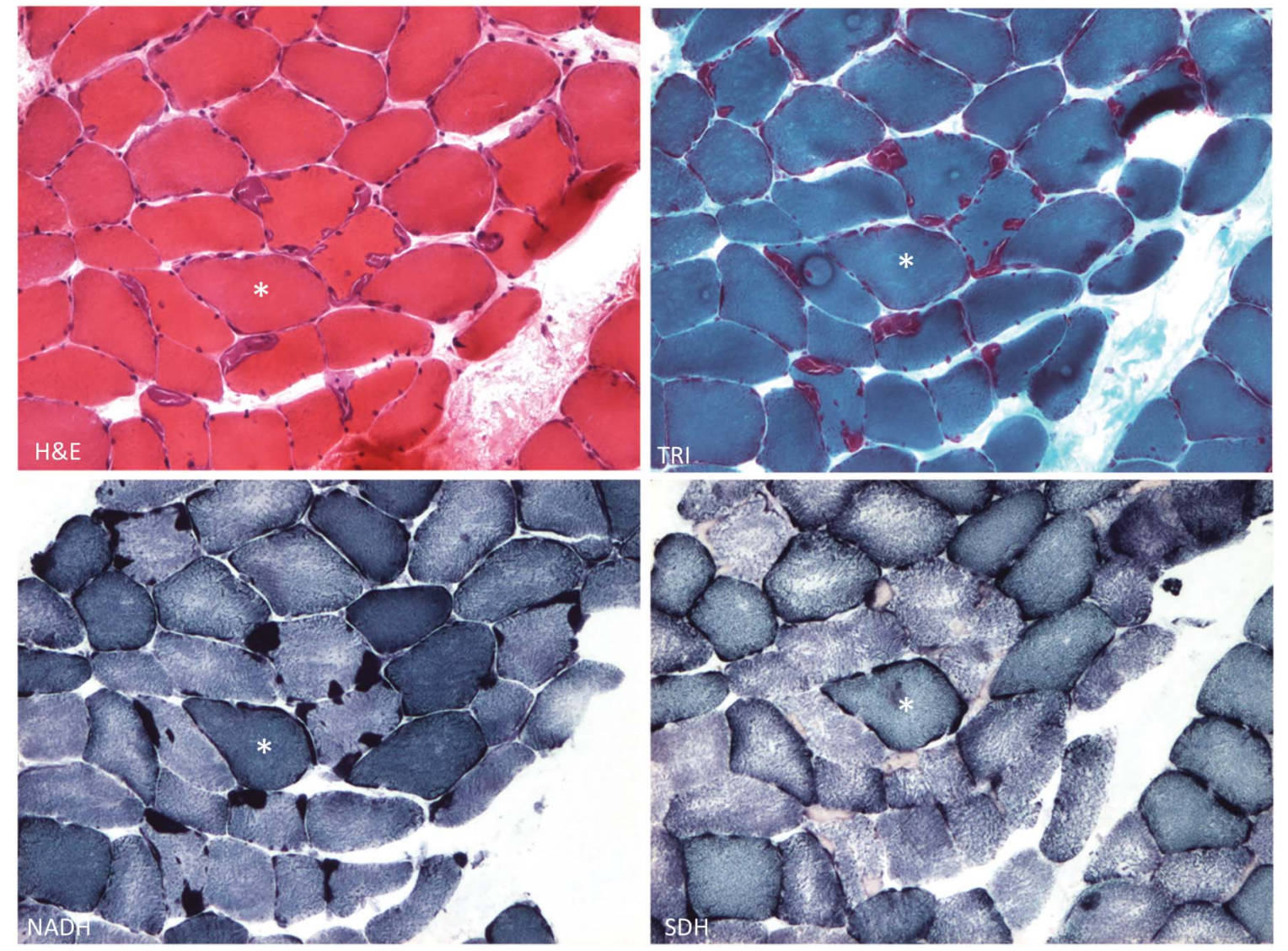

Serial cryosections $\left(^{*}\right.$ ) of patient 14 muscle biopsy (all panels original magnification $\times 20$ ). Several muscle fibers show vacuoles that appear basophilic in hematoxylin-eosin (H\&E), stained red in trichrome (TRI), stained dark blue in nicotinamide adenine dinucleotide (NADH), and did not react with succinicdehydrogenase (SDH), consistent with tubular aggregates.

2 of 22 patients had been diagnosed with a nonprogressive demyelinating encephalopathy, adding to the possible role of CASQ1 in CNS. However, brain MRI has not been performed systematically, and results are very preliminary. The clinical "red flags" for diagnosing CASQ1-related myopathy are proximal and axial muscle weakness with scapular winging, quadriceps hypotrophy, relative sparing of rectus anterior, brisk patellar reflexes, mild joint contractures, and high CK. The diagnosis of CASQ1 myopathy can also be suspected if muscle MRI is performed. An asymmetric age-dependent fibrofatty substitution of distal vastus lateralis, semitendinosus, gluteus maximus, and gastrocnemius medialis, with a relative sparing of vastus intermedius, sartorius, and proximal biceps femoris, was recurrent in our case series.

In 1 patient, left ventricular hypertrophy with minimal signs of fibrosis was observed. This patient presented with asymptomatic hyperCKemia, and he was not hypertensive. The association between heart disease and CASQ1 mutation has not been described to date, and it is known that CASQ1 is not highly expressed in heart. A single case observation does not allow us to rule out a chance association of an independent heart disease. Nevertheless, this finding indicates the necessity for careful monitoring of cardiac function in all CASQ1-mutated patients.

The most important clue for the diagnosis of CASQ1-related myopathy remains the histopathologic picture, which in p.Arg244Gly-mutated patients is always characterized by myopathic changes and vacuolar inclusions, optically empty in standard colorations. These vacuoles strongly react with antibodies against SR calcium-machinery proteins. It is known that CASQ1 regulates store-operated $\mathrm{Ca}^{2+}$ entry, interacting with STIM1 and inhibiting STIM1/ORAI1 interaction as demonstrated in HEK293 cells,,$^{30,34-36}$ and colocalizes with RYR1 at couplons ${ }^{36,37}$ where mechanical or allosteric communication among voltage sensors, $\mathrm{Ca}^{2+}$ release channels, and $\mathrm{Ca}^{2+}$ storage proteins takes place. ${ }^{37}$ These findings confirm that abnormal polymerization and aggregation of mutated CASQ1 lead not only to decreased $\mathrm{Ca}^{2+}$ release but also to an accumulation of all other interacting proteins, causing sarcomere destabilization and cell toxicity, as previously suggested. ${ }^{38,39}$ Moreover, it is interesting to note the histopathologic differences between p.Arg244Gly and p.Gly103Asp muscle. In fact, the latter showed vacuoles containing amorphous material, consistent with tubular 

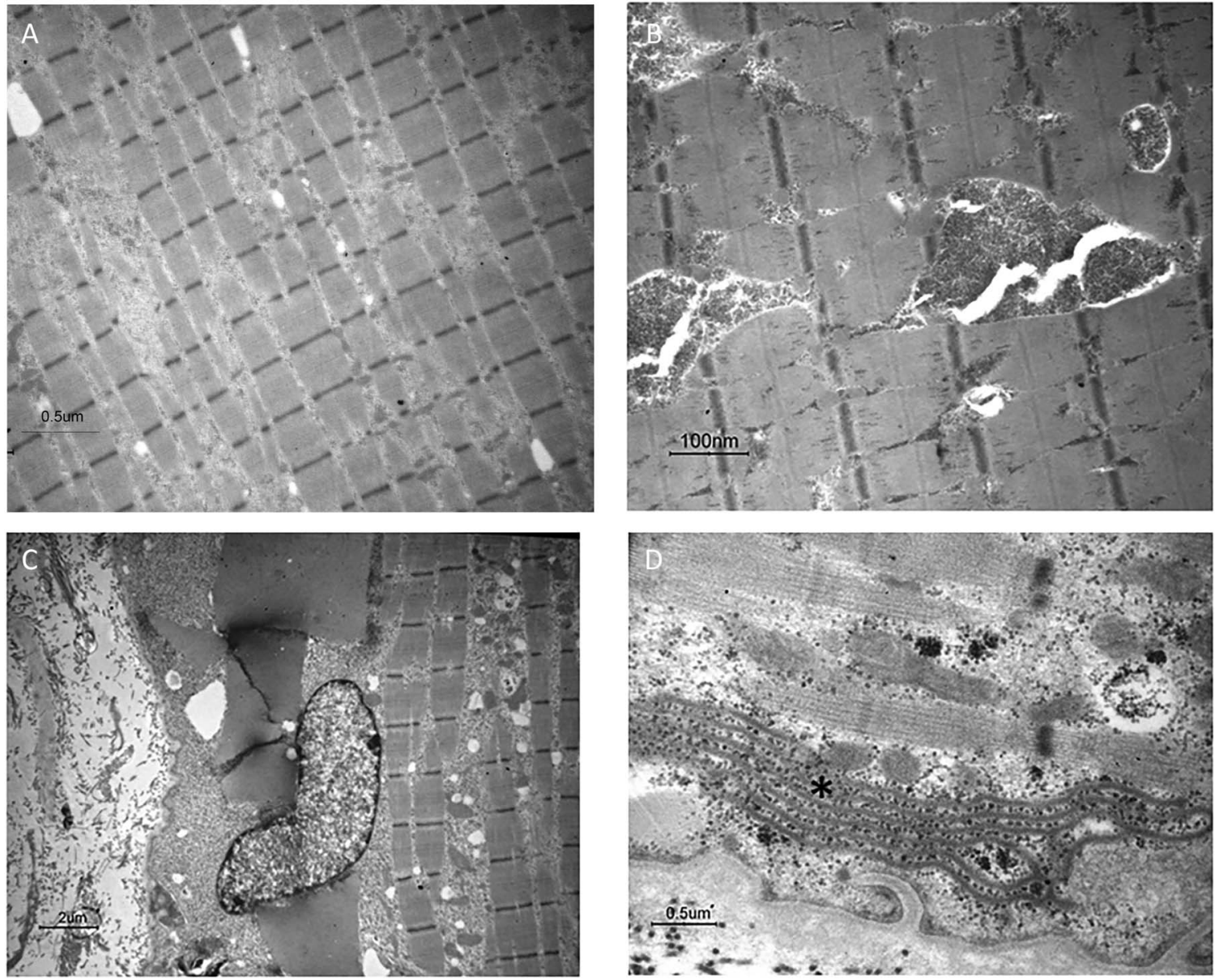

Scattered myofibrillar lysis area replaced by (B) a progressive accumulation of glycogen granules is shown. (C) Multiple inclusions, partially or entirely membrane bounded, sometimes gathered to constitute a conglomerate mass with a geometric shape in the myofiber sarcoplasm. The inclusions feature variable electron density from a light loosely textured fibrillar matrix to a homogeneous dark-appearing content. (D) Few tubular aggregates can be also detectable $(*)$ in the subsarcolemmal area.

aggregate pathology, as recently reported also for other CASQ1 mutations. ${ }^{8}$ Furthermore, the CASQ1-interacting proteins ORAI1, STIM1, RYR1 (data not shown), and SERCA1 appear to fill the vacuoles instead of decorating the edge. These results suggest that the effect of CASQ1 mutations on the interacting proteins differs according to the mutated residue and its functional consequences.

The slow evolution of the CASQ1 myopathy may be due to redundance of the complex molecular mechanisms regulating calcium homeostasis, which can compensate for the abnormal functioning of calsequestrin. However, these mechanisms need further investigation.

The triadopathies are emerging muscle diseases due to genetic defects in 1 of the components of the triad (RYR1, ORAI1, STIM1, CASQ1). ${ }^{2}$ These pathologies frequently present with overlapping phenotypic, molecular, and histopathologic characteristics that are being just recently clarified. Each gene may be associated with different clinical pictures, but similar histopathologic changes can be caused by mutations in different genes. For example, myalgia or rhabdomyolysis can be observed in CASQ1- but also RYR1- and ORAI1-related myopathies. ${ }^{40,41}$ In these myopathies, histopathologic features are different and characterized either by core-like structures on oxidative stains (RYR1) or by tubular aggregates (ORAI1, STIM1) and vacuolar inclusions (CASQ1). Our patient presenting the p.Gly103Asp mutation showed $\mathrm{NADH}$-TR-positive inclusion on muscle biopsy, confirming an overlap with tubular aggregate myopathies. ${ }^{7,8}$

In the light of our findings, the phenotypic spectrum of CASQ1-related myopathies appears expanded to include patients with severe muscle weakness, fibrofatty substitution, and a possible heart involvement. At the same time, its boundaries with other triadopathies and $\mathrm{Ca}^{2+}$-related myopathies appear more blurred, with many shared clinical and histopathologic features. 


\section{Author contributions}

C. Semplicini contributed to the design and conceptualization of the study, the selection and clinical evaluation of patients, the analysis and interpretation of data, and drafting the manuscript. C. Bertolin contributed to the analysis and interpretation of molecular/histopathologic data and drafting the manuscript. L. Bello contributed to the design and conceptualization of the study, the selection and clinical evaluation of patients, the analysis and interpretation of data, and drafting the manuscript. B. Pantic contributed to the analysis and interpretation of molecular/histopathologic data and drafting the manuscript. F. Guidolin contributed to the selection and clinical evaluation of patients, the analysis and interpretation of data, and revising the manuscript. S. Vianello and F. Catapano contributed to the analysis and interpretation of molecular/histopathologic data and revising the manuscript. I. Colombo contributed to the selection and clinical evaluation of patients, the analysis and interpretation of data, and revising the manuscript. M. Moggio and B.F. Gavassini contributed to the analysis and interpretation of molecular/histopathologic data and revising the manuscript. G. Cenacchi contributed to the analysis and interpretation of molecular/histopathologic data and drafting the manuscript. V. Papa contributed to the analysis and interpretation of molecular/histopathologic data and revising the manuscript. M. Previtero and C. Calore contributed to the selection and clinical evaluation of patients, the analysis and interpretation of data, and revising the manuscript. G. Sorarù contributed to the selection and clinical evaluation of patients and revising the manuscript. G. Minervini contributed to the analysis and interpretation of molecular/histopathologic data and drafting the manuscript. S.C.E. Tosatto contributed to the analysis and interpretation of molecular/histopathologic data and revising the manuscript. R. Stramare contributed to the analysis and interpretation of muscle MRI and revising the manuscript. E. Pegoraro contributed to the design and conceptualization of the study, the selection and clinical evaluation of patients, the analysis and interpretation of data, and drafting the manuscript.

\section{Study funding}

The authors acknowledge support from the Telethon BioBank (GTB12001D), the EuroBioBank network, and the Italian Ministry of Health (RF-2013-02359065), and a grant from the University of Padova (CPDA151054 to E.P.).

\section{Disclosure}

C. Semplicini, C. Bertolin, L. Bello, B. Pantic, F. Guidolin, S. Vianello, F. Catapano, I. Colombo, M. Moggio, B. Gavassini, G. Cenacchi, V. Papa, M. Previtero, C. Calore, G. Sorarù, G. Minervini, S. Tosatto, and R. Stramare report no disclosures relevant to the manuscript. E. Pegoraro reports personal fees from PTC Therapeutics, Santhera, and Sanofi Genzyme. Go to Neurology.org/N for full disclosures.

\section{Publication history}

Received by Neurology January 10, 2018. Accepted in final form July 17, 2018.

\section{References}

1. Rossi AE, Dirksen RT. Sarcoplasmic reticulum: the dynamic calcium governor of muscle. Muscle Nerve 2006;33:715-731.

2. Dowling JJ, Lawlor MW, Dirksen RT. Triadopathies: an emerging class of skeletal muscle diseases. Neurotherapeutics 2014;11:773-785.

3. Kawasaki T, Kasai M. Regulation of calcium channel in sarcoplasmic reticulum by calsequestrin. Biochem Biophys Res Commun 1994;199:1120-1127.

4. Lamboley CR, Murphy RM, Mckenna MJ, Lamb GD. Endogenous and maximal sarcoplasmic reticulum calcium content and calsequestrin expression in type I and type II human skeletal muscle fibres. J Physiol 2013;591:6053-6068.

5. Rossi D, Vezzani B, Galli L, et al. A mutation in the CASQ1 gene causes a vacuolar myopathy with accumulation of sarcoplasmic reticulum protein aggregates. Hum Mutat 2014;35:1163-1170.

6. Di Blasi C, Sansanelli S, Ruggieri A, et al. A CASQ1 founder mutation in three Italian families with protein aggregate myopathy and hyperCKaemia. J Med Genet 2015;52:617-626.

7. Böhm J, Lornage X, Chevessier F, et al. CASQ1 mutations impair calsequestrin polymerization and cause tubular aggregate myopathy. Acta Neuropathol 2018;135: 149-151.

8. Barone V, Del Re V, Gamberucci A, et al. Identification and characterization of three novel mutations in the CASQ1 gene in four patients with tubular aggregate myopathy. Hum Mutat 2017;38:1761-1773.

9. Consortium UniProt. Activities at the universal protein resource (UniProt). Nucleic Acids Res 2014;42:D191-D198.

10. Schneider A, Dessimoz C, Gonnet GH. OMA Browser: exploring orthologous relations across 352 complete genomes. Bioinformatics 2007;23:2180-2182.

11. Waterhouse AM, Procter JB, Martin DMA, Clamp M, Barton GJ. Jalview version 2: a multiple sequence alignment editor and analysis workbench. Bioinformatics 2009; 25:1189-1191.

12. Webb B, Sali A. Comparative protein structure modeling using MODELLER. Curr Protoc Bioinformatics. 2016;54:5.6.1-5.6.3.

13. Giollo M, Martin AJM, Walsh I, Ferrari C, Tosatto SCE. NeEMO: a method using residue interaction networks to improve prediction of protein stability upon mutation. BMC Genomics 2014;15(suppl 4):S7.

14. Cheng J, Randall A, Baldi P. Prediction of protein stability changes for single-site mutations using support vector machines. Proteins 2006;62:1125-1132.

15. Adzhubei IA, Schmidt S, Peshkin L, et al. A method and server for predicting damaging missense mutations. Nat Methods 2010;7:248-249.

16. Kumar P, Henikoff S, Ng PC. Predicting the effects of coding non-synonymous variants on protein function using the SIFT algorithm. Nat Protoc 2009;4:1073-1081.

17. Ferrer-Costa C, Orozco M, de la Cruz X. Sequence-based prediction of pathological mutations. Proteins 2004;57:811-819.

18. Yates CM, Filippis I, Kelley LA, Sternberg MJE. SuSPect: enhanced prediction of single amino acid variant (SAV) phenotype using network features. J Mol Biol 2014; 426:2692-2701.

19. Capriotti E, Fariselli P, Casadio R. I-Mutant2.0: predicting stability changes upon mutation from the protein sequence or structure. Nucleic Acids Res 2005;33: W306-W310.

20. Walsh I, Minervini G, Corazza A, Esposito G, Tosatto SCE, Fogolari F. Bluues server: electrostatic properties of wild-type and mutated protein structures. Bioinformatics 2012;28:2189-2190.

21. Pettersen EF, Goddard TD, Huang CC, et al. UCSF Chimera: a visualization system for exploratory research and analysis. J Comput Chem 2004;25:1605-1612.

22. Piovesan D, Walsh I, Minervini G, Tosatto SCE. FELLS: fast estimator of latent local structure. Bioinformatics 2017;33:1889-1891.

23. Piovesan D, Minervini G, Tosatto SCE. The RING 2.0 web server for high quality residue interaction networks. Nucleic Acids Res 2016;44:W367-W374.

24. Phillips JC, Braun R, Wang W, et al. Scalable molecular dynamics with NAMD. J Comput Chem 2005;26:1781-1802.

25. MacKerell AD, Bashford D, Bellott M, et al. All-atom empirical potential for molecular modeling and dynamics studies of proteins. J Phys Chem B 1998;102:3586-3616.

26. Hornbeck PV, Zhang B, Murray B, Kornhauser JM, Latham V, Skrzypek E. PhosphoSitePlus, 2014: mutations, PTMs and recalibrations. Nucleic Acids Res 2015;43: D512-D520.

27. Mercuri E, Pichiecchio A, Allsop J, Messina S, Pane M, Muntoni F. Muscle MRI in inherited neuromuscular disorders: past, present, and future. J Magn Reson Imaging 2007;25:433-440.

28. Tomelleri G, Palmucci L, Tonin P, et al. SERCA1 and calsequestrin storage myopathy: a new surplus protein myopathy. Brain J Neurol 2006;129:2085-2092.

29. Zhu Z, Zhou H, Yu X, et al. Potential regulatory role of calsequestrin in platelet $\mathrm{Ca}^{2+}$ homeostasis and its association with platelet hyperactivity in diabetes mellitus: role of reduced calsequestrin in diabetic platelets. J Thromb Haemost 2012; 10:116-124.

30. Wang Y, Xu L, Duan H, Pasek DA, Eu JP, Meissner G. Knocking down type 2 but not type 1 calsequestrin reduces calcium sequestration and release in $\mathrm{C} 2 \mathrm{C} 12$ skeletal muscle myotubes. J Biol Chem 2006;281:15572-15581.

31. Tissue expression of CASQ1-summary: the Human Protein Atlas [online]. Available at: proteinatlas.org/ENSG00000143318-CASQ1/tissue. Accessed June 21, 2018. 
32. Volpe P, Gorza L, Brini M, Sacchetto R, Ausoni S, Clegg DO. Expression of the calsequestrin gene in chicken cerebellum Purkinje neurons. Biochem J 1993;294: 487-490.

33. Weaver SA, Schaefer AL, Dixon WT. The effects of mutated skeletal ryanodine receptors on calreticulin and calsequestrin expression in the brain and pituitary gland of boars. Mol Brain Res 2000;75:46-53.

34. Zhao X, Min CK, Ko JK, et al. Increased store-operated Ca2+ entry in skeletal muscle with reduced calsequestrin-1 expression. Biophys J 2010;99:1556-1564.

35. Shin DW, Pan Z, Kim EK, et al. A retrograde signal from calsequestrin for the regulation of store-operated $\mathrm{Ca}^{2+}$ entry in skeletal muscle. J Biol Chem 2003;278: 3286-3292.

36. Beard NA, Dulhunty AF. C-terminal residues of skeletal muscle calsequestrin are essential for calcium binding and for skeletal ryanodine receptor inhibition. Skelet Muscle 2015;5:6.
37. Ríos E, Figueroa L, Manno C, Kraeva N, Riazi S. The couplonopathies: a comparative approach to a class of diseases of skeletal and cardiac muscle. J Gen Physiol 2015;145: 459-474.

38. D'Adamo MC, Sforna L, Visentin S, et al. A calsequestrin-1 mutation associated with a skeletal muscle disease alters sarcoplasmic $\mathrm{Ca}^{2+}$ release. PLoS One 2016;11: e0155516.

39. Lewis KM, Ronish LA, Ríos E, Kang C. Characterization of two human skeletal calsequestrin mutants implicated in malignant hyperthermia and vacuolar aggregate myopathy. J Biol Chem 2015;290:28665-28674.

40. Dlamini N, Voermans NC, Lillis S, et al. Mutations in RYR1 are a common cause of exertional myalgia and rhabdomyolysis. Neuromuscul Disord 2013;23:540-548.

41. Endo Y, Noguchi S, Hara Y, et al. Dominant mutations in ORAIl cause tubular aggregate myopathy with hypocalcemia via constitutive activation of store-operated Ca2+ channels. Hum Mol Genet 2015;24:637-648. 